\title{
VALIDITY OF COMPUTED TOMOGRAPHIC STUDY IN PREOPERATIVE STAGING OF GASTRIC CANCER WITH REFERENCE TO SURGICAL AND HISTOPATHOLOGIC RESULTS
}

\author{
Rizana Sooraj ${ }^{1}$, Tixon Thomas ${ }^{2}$, Srikanth Moorthy33, Sreekumar Karumathil Pullara ${ }^{4}$, Ramiah Rajesh Kannan ${ }^{5}$, Nirmal Kumar Prabhu ${ }^{6}$, \\ Sunil Patel7, Vinayak Jayaram ${ }^{8}$

\begin{abstract}
1 Junior Resident, Department of Radiology, Amrita Institute of Medical Sciences and Research Institute. ${ }^{2}$ Assistant Professor, Department of Radiology, Amrita Institute of Medical Sciences and Research Institute.

${ }^{3}$ Professor, Department of Radiology, Amrita Institute of Medical Sciences and Research Institute.

${ }^{4}$ Professor, Department of Radiology, Amrita Institute of Medical Sciences and Research Institute.

${ }^{5}$ Associate Professor, Department of Radiology, Amrita Institute of Medical Sciences and Research Institute.

${ }^{6}$ Associate Professor, Department of Radiology, Amrita Institute of Medical Sciences and Research Institute.

${ }_{7}$ Senior Resident, Department of Radiology, Amrita Institute of Medical Sciences and Research Institute.
\end{abstract} \\ ${ }^{8}$ Senior Resident, Department of Radiology, Amrita Institute of Medical Sciences and Research Institute.
}

ABSTRACT

\section{BACKGROUND}

Gastric carcinoma represents the most common gastric neoplasm accounting for $95 \%$ of all gastric tumours and represents a group of aggressive malignancy. Although highly aggressive, the early gastric cancer shows a better prognosis with a 5-year survival rate of more than $90 \%$. There is a wide variation of the prognosis varying from less than $3 \%$ to $90 \%$ in early gastric cancers. Early diagnosis and accurate staging are therefore very crucial for the choice of an accurate therapeutic approach and also for the good survival rate.

This study aims at evaluating the validity of MDCT in staging gastric cancer.

\section{MATERIALS AND METHODS}

Between May 2014 and September 2016, 35 patients (27 males, 8 females), with mean age of 63 years were evaluated. CT was performed using 16 slice and 64 slice CT and Tumour staging and nodal staging was done according to TNM staging put forward by AJCC 7th edition. All the stages were compared with the gold standard histopathological staging. Site and extent of the lesion and surgery done were also assessed.

\section{RESULTS}

The comparison of tumour and nodal staging to histopathological staging was statistically done by McNemar test, and all the stages of the malignancy showed statistical agreement between the two. The sensitivity, specificity, accuracy and $\mathrm{p}$ value of all the $\mathrm{T}$ stages and $\mathrm{N}$ stages were the statistical parameters assessed. The sensitivity of early gastric cancer (T1 and T2) was comparatively lower than advanced cases (T3 and T4). The sensitivity of staging T1 lesion was 55\% whereas it was $93 \%$ in staging T3 lesions. The sensitivity of nodal staging was about $70 \%$ in all the stages.

\section{CONCLUSION}

CT as a modality in staging gastric cancer helps staging the advanced gastric cancer more accurately than early gastric cancer. Along with the tumour and nodal staging it can also give the details of distant organ metastasis. It can be used in conjunction with other endoscopic methods for accurate preoperative staging.

\section{KEYWORDS}

Gastric, Cancer Staging, Histopathology and CECT Correlation.

HOW TO CITE THIS ARTICLE: Sooraj R, Thomas T, Moorthy S, et al. Validity of computed tomographic study in preoperative staging of gastric cancer with reference to surgical and histopathologic results. J. Evolution Med. Dent. Sci. 2017;6(11):891-896, DOI: $10.14260 / \mathrm{Jemds} / 2017 / 191$

\section{BACKGROUND}

Gastric carcinoma represents the most common gastric neoplasm accounting for $95 \%$ of all gastric tumours.(1) and represents a group of aggressive malignancy.

Financial or Other, Competing Interest: None.

Submission 12-01-2017, Peer Review 24-01-2017,

Acceptance 28-01-2017, Published 06-02-2017.

Corresponding Author:

Rizana Sooraj,

Department of Radiology,

Amrita Institute of Medical Sciences and Research Institute,

Ponekkara, Kochi-682041,

Kerala.

E-mail: rizbubly@gmail.com

DOI: $10.14260 /$ jemds $/ 2017 / 191$
Although highly aggressive, the early gastric cancer shows a better prognosis with a 5-year survival rate of more than $90 \%$. There is a wide variation of the prognosis varying from less than $3 \%$ to $90 \%$ in early gastric cancers. (2) Early diagnosis and accurate staging are therefore very crucial for the choice of an accurate therapeutic approach and also for the good survival rate.(3)

Imaging gastric cancer has attracted intense interest and been the subject of numerous research studies over the past several years due to high mortality rate in advanced disease, early detection of the tumour provides a very good prognosis, early detection of the tumour is giving way to non-invasive treatment modalities.

In current era, there is a lot of importance towards minimally invasive methods for diagnosis and management of gastric cancer, the mainstay of diagnosis being a computed 
tomographic staging along with endoscopic biopsy. Contrast enhanced computed tomography (CECT) along with the local disease staging gives an overview of the disease progression to other organs and lymph nodes making it the modality of choice for diagnosis.

The stomach is the widest part of the alimentary tract and lies between the oesophagus and the duodenum, the stomach is divided by arbitrary lines drawn on its external surface into a fundus, body, pyloric antrum and pylorus. Histologically, the stomach wall is divided into mucosa, submucosa, muscularis propria and serosa.

The main risk factors of the malignancy include dietary habits, H. pylori infection, atrophic gastritis, pernicious anaemia and rarely because of gastric polyp and Menetrier's disease.(4-7) Gastric carcinoma is twice as common in men as in women. However, carcinoma of the cardia has a much greater predilection for men (7:1) than carcinoma elsewhere in the stomach.(8) The explanation for this discrepancy is unclear. The most common presenting findings include epigastric pain, bloating, early satiety, nausea, vomiting, dysphagia, anorexia, weight loss and signs or symptoms of upper gastrointestinal bleeding, such as haematemesis, melaena and iron-deficiency anaemia.(9) Early GC is defined as adenocarcinoma limited to the mucosa and submucosa of the stomach, irrespective of lymph node status. Late GC defined as a gastric carcinoma that has invaded the muscle wall. The routes of spread can be either direct, haematogenous, lymphatic or intraperitoneally. Staging of gastric cancer is essential for providing the best available treatment option. Gastric cancer is staged according to AJCC $7_{\text {th }}$ edition. According to AJCC, the tumour is staged as T1, T2, $\mathrm{T} 3$ and T4 and nodes are staged as N0, N1, N2 and N3 stages. Patients presenting with the above-mentioned symptoms and those with multiple risk factors for gastric carcinoma require further workup. Oesophagogastroduodenoscopy (EGD) is the diagnostic imaging procedure of choice in the work-up of gastric carcinoma.(10) However, a double-contrast barium swallow, a cost-conscious, noninvasive and readily available study, may be the initial step for the physician to determine if a gastric lesion is present and whether the lesion is benign or malignant.(11)

TNM classification of gastric carcinomas and the relevant imaging findings: Owing to recent advances in CT technology, we have been able to visualise early carcinomas and to stage tumours with considerable accuracy, with the use of appropriate contrast technique and effervescent agent or water. Recent CT with conventional transverse images, multiplanar reconstruction (MPR) images and virtual endoscopy can detect gastric carcinomas efficiently with the detection rates of $91 \%, 96 \%$ and $98 \%$, respectively.(12) Gastric carcinomas appear as a focal area of mural thickening with or without ulceration, as a polypoid lesion, or as generalised mural thickening. Lesions occurring in the antrum, in the body, and in the fundus comprise $30 \%$ of all gastric carcinomas respectively, and the remaining $10 \%$ involve the whole stomach.(2) CT criteria for $\mathrm{T}$ staging of gastric carcinoma is as follows.(12) $\mathrm{T} 1$ lesion = focal thickening of the inner layer, almost well enhanced, and has visible low attenuation-strip outer layer of gastric wall and clear fat plane around tumour. T2 lesion = focal or diffuse thickening of the wall with transmural enhancement, almost well enhanced, and has smooth outer wall border and clear fat plane around tumour. T3 lesion = transmural tumour with irregular or nodular outer border and/or perigastric fat infiltration. $\mathrm{T} 4$ lesion = Obliteration of fat plane between gastric tumour and adjacent organ or invasion of adjacent organ. Accuracy of CT in T-staging with transverse images only is $73 \%$, but it rises to $89 \%$ with the use of MPR.(12) Therefore, it is important to perform appropriate reconstruction techniques in CT diagnosis of gastric carcinoma.

$\mathrm{T} 1$ tumours are classified into $\mathrm{T} 1 \mathrm{a}$ and $\mathrm{T} 1 \mathrm{~b}$ tumours; a T1a lesion stay within the mucosal layer, while a T1b lesion stay within the submucosal layer. A T2 tumour infiltrates into the muscularis propria layer and stays within the layer. A T3 tumour extends over the muscularis propria layer, but its border stays within the sub- serosal layer. T4 tumours are classified into T4a and T4b tumours. A T4a lesion invades the serosa, exposing its surface to the peritoneal cavity in many cases. The tumour is classified as T4b when it invades the adjacent organs, such as the transverse colon, pancreas, spleen, liver and the diaphragm. Signet-ring cell carcinoma, often found at T4a stage, usually manifests as a scirrhous tumour, and appears as diffuse thickening of the gastric wall with obliteration of gastric folds, usually extending from the antrum into the body and fundus.(2) $\mathrm{T} 4 \mathrm{~b}$ tumour requires resection of adjacent organs with the primary tumour, and discrimination of T4a tumours from "T4b-looking tumour" is an important function of preoperative imaging. An advanced tumour can be recognised as T4a when the fat plane between the tumour and the adjacent organ is visualised, or when the fat plane is invisible or compressed by the tumour, the tumour is considered to be T4a if it has a clear and smooth border. MPR in appropriate plane is especially effective in differentiating between $\mathrm{T} 4 \mathrm{a}$ and $\mathrm{T} 4 \mathrm{~b}$; MPR is reported to improve the specificity without compensation in sensitivity in diagnosis of invasion into the transverse colon or mesocolon and the pancreas. ${ }^{(13)}$

\section{MATERIALS AND METHODS}

After obtaining approval from the Thesis Protocol Review Committee (Scientific, Ethical \& Financial), Amrita Institute of Medical Sciences the cross sectional observational study was carried out

My study included, Suspected cases of carcinoma stomach undergoing CECT followed by surgical resection and histopathological staging endoscopically detected cases of carcinoma stomach undergoing CECT followed by surgical resection and histopathological staging and incidentally detected stomach lesions in patients undergoing CECT for other causes followed by surgical resection and histopathological staging. Our study excluded patients who were not willing to undergo CECT surgical and histopathological staging.

\section{Sample Size}

Based on the accuracy of CT staging of gastric cancer with respect to histopathological outcome observed in an earlier publication by (FD Elia, Zingeralli, D Palli, M Grami on Hydrodynamic CT preoperative staging of gastric cancer : correlation with pathological findings) and with $20 \%$ allowable error and 95\% confidence minimum sample size comes to 30 .

CECT Technique and interpreting the image: First of all 
before computed tomographic study we made sure serum creatinine was within normal limits $(<1.4 \mathrm{mg} / \mathrm{dL})$, all the patients were fasting for 4 hours prior to the study. Stomach was adequately distended using $40 \mathrm{ml}$ of contrast of Gastrografin (1 mL of the solution contains $100 \mathrm{~mL}$ diatrizoate sodium $+660 \mathrm{mg}$ meglumine amidotrizoate) diluted in about 1 litre of water and had over half an hour. Apart from oral contrast, intravenous iodinated contrast, Omnipaque (Iohexol 350) of about $80 \mathrm{~mL}$ along with $20 \mathrm{~mL}$ of saline was given at about $2.3 \mathrm{ml} / \mathrm{sec}$. Initially an upper arterial phase at 35 seconds was taken including base of lung field and kidneys and a venous phase was taken at 70 seconds including the basal lung fields and till the pubic symphysis. Images were taken in supine position. The images were reconstructed in axial and coronal planes at $5 \mathrm{~mm}$ and $3 \mathrm{~mm}$ slice thicknesses respectively. The cases were done in either 16 slices or 64 slices CT scanners with no difference in abovementioned CT protocol. Images were checked for artefacts and adequate coverage and were of sufficient quality for evaluation. Computed tomography axial images of $1 \mathrm{~mm}$ slice thickness and reconstructions were examined for diagnosis on the workstation. Bi-dimensional multi-planar reconstructions on coronal and sagittal planes were examined as well to make the appropriate diagnosis. On CT images, the gastric wall is observed as three layers: wellenhancing mucosa, submucosa as a low attenuated stripe, and musculoserosal layers of slightly elevated attenuation. Gastric cancers manifest as having enhancing wall thickening on $\mathrm{CT}$, and the destruction of normal gastric wall structures can suggest the possible depth of invasion. For staging the malignancy we followed AJCC $7^{\text {th }}$ manual.

$\mathrm{T} 1$ tumours are sub-staged as $\mathrm{T} 1 \mathrm{a}$ and $\mathrm{T} 1 \mathrm{~b}$ : a stage $\mathrm{T} 1 \mathrm{a}$ tumour is confined to the mucosa, and T1b tumours have invaded the submucosa. On MDCT images, T1a tumours are usually not visible, and T1b tumours more frequently show mucosal thickening and enhancement. To differentiate between T1b and T2, T1b tumours show a low-attenuated stripe at the base of the tumour, which suggests a submucosal layer, while T2 tumours show loss of a low-attenuated stripe, which indicates involvement of the entire submucosal layer. T3 tumours have subserosal invasion, and discrimination between a gastric mass and the outer layer is visibly impossible, and smooth outer margin of the outer layer or a few small linear strandings in the perigastric fat plane can be noted. Stage T4a tumours also demonstrate serosal involvement, which makes differentiating between T3 and T4a using MDCT very difficult (the gastric serosa is not delineated on CT images). Stage T4b tumours show direct extension into an adjacent organ or structure and show obliteration of the fat plane between the gastric mass and adjacent organs.

Lymph nodal staging.(12-14) Malignant lymph nodes are those with short axis diameters of $6-8 \mathrm{~mm}$ for perigastric lymph nodes, other criteria used were like roundness, central necrosis, heterogenous enhancement, loss of fatty hilum, marked enhancement $(>80 \mathrm{HU})$ and clustering of more than three lymph nodes. These patients then underwent surgical resection followed by histopathological staging. The maximum time duration allowed between the CT and surgery was 1 month.

\section{Data analysis Plan and Statistical Tests}

Validity parameters namely sensitivity, specificity, accuracy and positive predictive/negative predictive value was computed for MDCT with respect to histopathology.

Statistical analyses were performed using IBM SPSS version 20.0. Categorical variables are presented using frequency and percentage. To find the statistical significant agreement of $\mathrm{T}$ stage and $\mathrm{N}$ stage between $\mathrm{CT}$ and HPR, McNemar's test was used.

\section{RESULTS}

In our study, there were 35 cases included, and the age group varied between 25 to 78 years with mean age of 63 years (Table -1, Figure-1). Out of the 35 patients, 27 were male and 8 were female (Table -2, Figure-2). Out of the 35 patients, according to our results 6 patients were in T1 stage, 5 in T2 stage, 18 in T3 stage and 6 were in T4 stage respectively according to CT (Table 3). In our study according to CT, 14 cases were in $\mathrm{N} 0$ and $\mathrm{N} 1$ stage (14patients), and least was in N2 stage (7 patients) (Table 4). Most of the included cases underwent total gastrectomy $(16 / 35)$, followed by subtotal gastrectomy $(14 / 35)$.

All the $\mathrm{T}$ staging and $\mathrm{N}$ staging according to CECT and HPR was concordant according to McNemar's test. In our study, out of the 35 patients there were 5 lesions detected as T2 lesions according to CT and out of them, 4 lesions were T1 lesions and the other one was a histopathologically proven $\mathrm{T} 3$ lesion (Appendix -1). In our study, out of 35 patients, there were 18 patients who were detected to have T3 lesion and out of which 15 were histopathologically proved, and the sensitivity, specificity, positive predictive value and negative predictive value and accuracy were 93.8\%, 84.2\%, 83.3\%, $94.1 \%$ and $88 \%$ respectively (Appendix- 2). In our study, there were 6 patients who were detected to have T4 lesions and out of which 6 were histopathologically proved, and the sensitivity, specificity, positive predictive value and negative predictive value and accuracy were $66.7 \%, 100 \%, 100 \%$, 89.7\% and 91\% respectively (Appendix -3 ).

The $\mathrm{p}$ value of $\mathrm{T} 4$ staging was 0.25 . In our study, out of 35 patients, there were 14 patients who were detected to have N0 staging and out of which 11 were histopathologically proved, and the sensitivity, specificity, positive predictive value, negative predictive value and accuracy were $73.3 \%$, $85 \%, 78.6 \%, 81 \%$ and $80 \%$ respectively (Appendix-4). In our study, there were 14 patients who were detected to be in $\mathrm{N} 1$ stage according to CT and out of which 11 were histopathologically proved, and the sensitivity, specificity, positive predictive value and negative predictive value and accuracy were 72.7\%, 75\%, 57.1\%, 85.7 and $74 \%$ (Appendix -5).

\begin{tabular}{|c|c|}
\hline $\begin{array}{c}\text { Age Group } \\
\text { (Years) }\end{array}$ & $\begin{array}{c}\text { Number } \\
\text { (Frequency) }\end{array}$ \\
\hline$<40$ & 2 \\
\hline $40-50$ & 2 \\
\hline $50-60$ & 4 \\
\hline \multicolumn{2}{|c|}{ Table 1. Showing the Distribution of } \\
Study Population among Various Age Groups \\
\hline
\end{tabular}




\begin{tabular}{|c|c|c|}
\hline Sex & Gender Distribution & $\mathbf{( \% )}$ \\
\hline Male & 27 & 77.1 \\
\hline Female & 8 & 22.9 \\
\hline Total & $\mathbf{3 5}$ & $\mathbf{1 0 0}$ \\
\hline \multicolumn{2}{|c|}{$\begin{array}{c}\text { Table 2. Showing Gender Wise } \\
\text { Distribution of the Study Population }\end{array}$} \\
\hline
\end{tabular}

\begin{tabular}{|c|c|}
\hline CT- T Staging & Number of Cases \\
\hline T1 & 6 \\
\hline T2 & 5 \\
\hline T3 & 18 \\
\hline T4 & 6 \\
\hline $\begin{array}{c}\text { Table 3. Showing the breakup of Patients to } \\
\text { different T Stages According to CT Evaluation }\end{array}$ \\
\hline
\end{tabular}

\begin{tabular}{|c|c|}
\hline CT-N Staging & Number of Cases \\
\hline N0 & 14 \\
\hline N1 & 14 \\
\hline N2 & 7 \\
\hline \multicolumn{2}{|c|}{$\begin{array}{c}\text { Table 4. Showing the breakup of Patients } \\
\text { to different N Stages According to CT }\end{array}$} \\
\hline
\end{tabular}

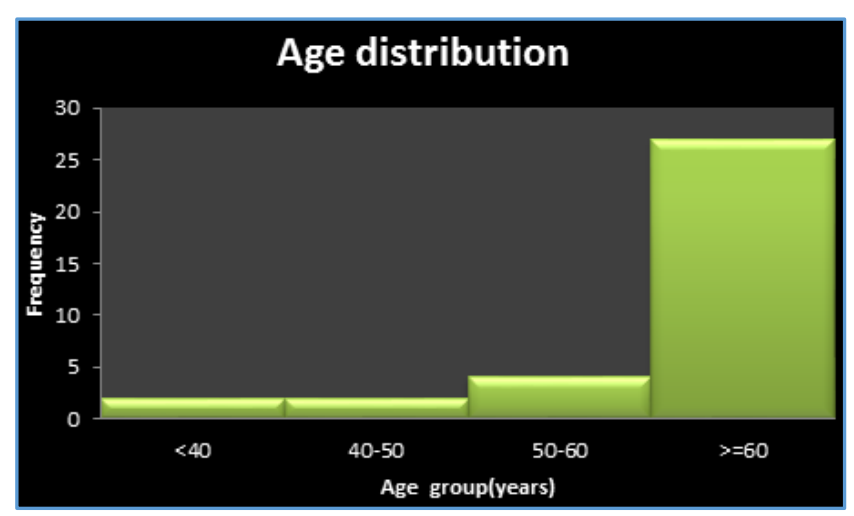

Figure 1. Showing Age Distribution of Our Study

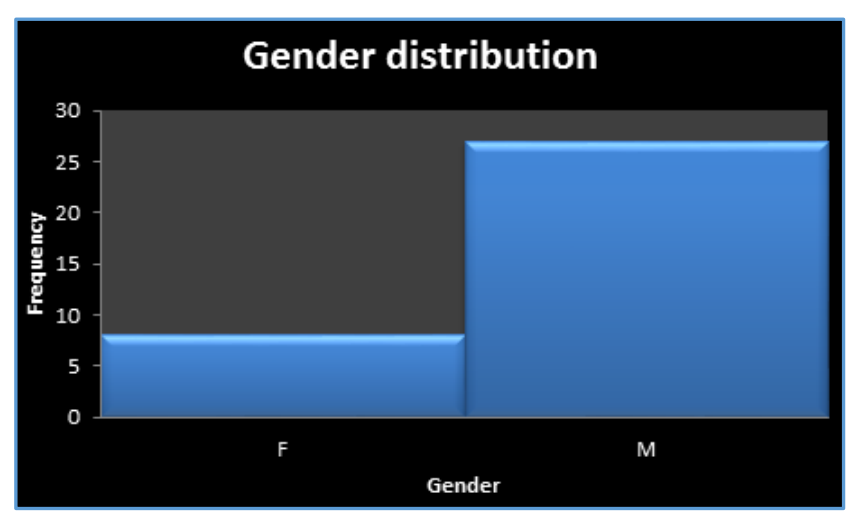

Figure 2. Showing the Gender Distribution of Our Study

\begin{tabular}{|c|c|c|c|}
\hline \multirow{2}{*}{ CT } & \multicolumn{2}{|c|}{ Histopathology } & \multirow{2}{*}{ p Value } \\
\cline { 2 - 3 } & Yes & No & \\
\cline { 2 - 3 } & $\mathbf{n = 9}$ & $\mathbf{n = 2 6}$ & \multirow{2}{*}{0.38} \\
\hline Yes (6) & $5(55.6)$ & $1(3.8 \%)$ & \\
\hline No (29) & $4(44.9)$ & $25(96.2)$ & \\
\hline \multicolumn{3}{|c|}{$\begin{array}{c}\text { Appendix 1. Depicting Comparison } \\
\text { of CT and HPR in T1 Staging }\end{array}$} \\
\hline
\end{tabular}

\begin{tabular}{|c|c|c|c|}
\hline \multirow[b]{2}{*}{ CT } & \multicolumn{2}{|c|}{ Histopathology } & \multirow[b]{2}{*}{ p Value } \\
\hline & $\begin{array}{c}\text { Yes } \\
n=16(\%)\end{array}$ & $\begin{array}{c}\text { No } \\
\mathrm{n}=19(\%)\end{array}$ & \\
\hline Yes (18) & $15(93.8)$ & $3(15.8)$ & \multirow{2}{*}{0.63} \\
\hline No (17) & $1(6.2)$ & $16(84.2)$ & \\
\hline \multicolumn{4}{|c|}{$\begin{array}{l}\text { Appendix 2. Depicting Comparison } \\
\text { of CT and HPR in T3 Staging }\end{array}$} \\
\hline \multirow[b]{2}{*}{ CT } & \multicolumn{2}{|c|}{ Histopathology } & \multirow[b]{2}{*}{ p Value } \\
\hline & $\begin{array}{c}\text { Yes } \\
n=9(\%)\end{array}$ & $\begin{array}{c}\text { No } \\
n=26(\%)\end{array}$ & \\
\hline Yes (6) & $6(66.7)$ & 0 & \multirow{2}{*}{0.25} \\
\hline No (29) & $3(33.3)$ & $26(100)$ & \\
\hline & $\begin{array}{l}\text { endix 3. De } \\
\text { of CT and } \mathrm{H}\end{array}$ & $\begin{array}{l}\text { g Comparis } \\
\text { T4 Staging }\end{array}$ & \\
\hline
\end{tabular}

\begin{tabular}{|c|c|c|c|}
\hline \multirow{2}{*}{ CT } & \multicolumn{2}{|c|}{ Histopathology } & \multirow{2}{*}{ P Value } \\
\cline { 2 - 3 } & $\begin{array}{c}\text { Yes } \\
\mathbf{n = 1 5}(\%)\end{array}$ & $\begin{array}{c}\text { No } \\
\mathbf{n = 2 0}(\%)\end{array}$ & \\
\hline Yes (14) & $11(73.3)$ & $3(15)$ & \multirow{2}{*}{1} \\
\hline No (21) & $4(26.7)$ & $17(85)$ & \\
\hline \multicolumn{3}{|c|}{$\begin{array}{c}\text { Appendix 4. Depicting Comparison } \\
\text { of CT and HPR in NO-Staging }\end{array}$} \\
\hline
\end{tabular}

\begin{tabular}{|c|c|c|c|}
\hline \multirow{2}{*}{ CT } & \multicolumn{2}{|c|}{ Histopathology } & \multirow{2}{*}{ p Value } \\
\cline { 2 - 3 } & $\begin{array}{c}\text { Yes } \\
\mathbf{n = 1 1}(\%)\end{array}$ & $\begin{array}{c}\text { No } \\
\mathbf{n}=\mathbf{2 4}(\mathbf{\%})\end{array}$ & \\
\hline Yes (14) & $8(72.7)$ & $6(25)$ & \multirow{2}{*}{0.51} \\
\hline No (21) & $3(27.3)$ & $18(75)$ & \\
\hline \multicolumn{3}{|c|}{$\begin{array}{c}\text { Appendix 5. Depicting Comparison } \\
\text { of CT and HPR in N1-Staging }\end{array}$} \\
\hline
\end{tabular}

\section{DISCUSSION}

Gastric cancer is one of the most common and fatal cancers. Although its prevalence is decreasing in Western countries, gastric cancer remains the second most common cause of cancer-related death. Gastric cancer is more common in Asian countries, particularly China, Japan, and South Korea.(1,2) The tumour-node-metastasis staging system is the generally accepted staging system for predicting the prognosis of patients with gastric cancer. Multidetector Computed tomography (MDCT) is a widely accepted imaging modality for the preoperative staging of gastric cancer that can simultaneously assess locoregional staging, including the gastric mass, regional lymph nodes, and distant metastasis. The diagnostic performance of MDCT for T- and N-staging has been improved by the technical development of isotropic imaging and 3D reformation.

Staging of gastric carcinoma is one of the most important step in managing the disease and due to various available options to treat and potentially cure it, accurate staging becomes even more critical. The choice of treatment for patients with gastric cancer relies on the TNM stage of the disease. There are several available treatment options, including surgery, chemotherapy and radiotherapy. Among the treatment options, complete surgical resection of the gastric cancer is generally considered the only effective curative treatment.(15,16) A lesion limited to the mucosa and the superficial submucosa above the muscularis propria can be accessed by endoscopy, such as EMR or endoscopic submucosal dissection (ESD). Endoscopic treatments can only be curative when there is no lymph node metastasis at the time of treatment. The T3/T4 lesions, on the other hand, or those with lymph nodal involvement generally undergo 
neoadjuvant chemotherapy or radiotherapy with surgery. The cases with metastasis generally remain inoperable and surgical intervention in such cases is limited to palliation. In our study, a total of 35 cases were evaluated using standardised CT protocol. Of these, 16 cases were suspected to be a gastric malignancy on endoscopy prior to the CECT evaluation and remaining 19 cases were incidentally detected to have a gastric wall lesion while being evaluated for upper GI symptoms or other cause. The demographic matching for sex was comparable to other studies which showed male predominance. In our study, 27 cases (77\%) were male and 8 were female which is very similar to data published by Yeole $^{(3)}$ and Jemal,(17,18) which had shown a male predominance with a male to female ratio of $2.3: 1$. The mean age observed in our study was 63 years and age of the patients varied from 25 to 78 years which is also near similar to the trend observed in Population Based Cancer Registry of Kollam and Thiruvananthapuram. ${ }^{19,20)}$ The cases were randomly allocated to 16 slice and 64 slice MDCT. The images obtained in either of the machines are comparable and of sufficient quality. We did not come across any study which had compared the sensitivity, specificity or accuracy on two different MDCTs. On CT images, the gastric wall is observed as three layers: well-enhancing mucosa, submucosa as a low attenuated stripe, and musculoserosal layers of slightly elevated attenuation.(21) On MDCT images, T1a tumours are usually not visible, and T1b tumours frequently show mucosal thickening and enhancement.(22,23) Differentiation between T1a and T1b lesions is best done with EUS.(24) Management of T1a and T1b lesions is essentially similar in the absence of nodal involvement. With $6 \mathrm{~mm}$ cut-off as thickness for abnormal gastric wall thickening, a total of $5 \mathrm{~T} 1$ lesions were accurately detected on MDCT. Sensitivity, specificity and accuracy for T1 lesions was 55.6\%, 96.2\% and $85.7 \%$ which implies that CT has a good positive predictive value in detecting $\mathrm{T} 1$ lesions. One of the cases with pyloric mucosal thickening, which was staged as T1 actually turned out to be tumour free. According to CT, 3 cases were over staged to $\mathrm{T} 2$ lesions because the low attenuating mucosal stripe was not visualised and all those lesions histopathologically proved to be T1b lesions. Similar study by Chen et al had shown sensitivity, specificity and accuracy of $78 \%, 100 \%$ and $96 \%$ respectively using MPR technique. The accuracy and specificity of our study was similar to this study. The lower sensitivity of our study may be because of the positive contrast used. To differentiate between T1 and T2, T1 tumours show a low attenuated stripe at the base of the tumour, which suggests an intact submucosal layer, while T2 tumours show loss of the low attenuating submucosal stripe. In our study 5 , T2 lesions were detected on CT. Of which 4 lesions were $\mathrm{T} 1$ lesions and one was proven to be T3 which could have been due to microscopic fat infiltration. In addition, the differentiation of perigastric infiltration from gastric cancer and perigastric inflammation or fibrosis on CT images can be difficult thus T2 tumours could be over-staged as T3 tumours or T4 tumours.(24) For T3 lesions the sensitivity, specificity and accuracy were 94\%, 84\% \& 89\% respectively. A total of $18 \mathrm{~T} 3$ tumours were detected in our study of which 3 were upgraded to T4 on histopathology. T3 tumours have subserosal invasion, and smooth outer margin of the outer layer or a few small linear strandings in the perigastric fat representing inflammatory changes. For T4 lesions, sensitivity, specificity and accuracy were $67 \%, 100 \%$ \& 91\% respectively. Chen et al Shimizu et al(25) and Heberman et al(3) had similarly reported accuracy between $93 \%$ to $98 \%$ for $\mathrm{T} 3 / \mathrm{T} 4$ lesions. T4a tumours also demonstrate serosal involvement, which makes differentiation between T3 and T4a using MDCT difficult. In addition, the amount of adipose tissue in the subserosal area varies from person to person. T4a tumours frequently show micronodules or dense, band-like stranding in the perigastric area. A total of 6, T4 lesions were detected using CT in our study all of which were T4a. One lesion on histopathological staging showed adjacent small bowel infiltration (T4b). To determine the optimal treatment method for each patient, accurate $\mathrm{N}$-staging is as important as $\mathrm{T}$-staging. $\mathrm{N}$-staging provides critical information that is needed to appropriately predict a patient's prognosis. For EMR or endoscopic submucosal dissection, N0 should be confirmed using EUS or MDCT. Additionally, as extensive lymphadenopathy detected surgically is known to be associated with higher morbidity and mortality, aggressive surgical procedures should be avoided for the patients with extensive lymphadenopathy. The results of studies evaluating the accuracy of MDCT in Nstaging are somewhat disappointing. According to the metaanalysis by Kwee et al,(26) the sensitivity and specificity of MDCT N-staging varied between $62.5 \%$ and $91.9 \%$ and $50.0 \%$ and $87.9 \%$, respectively. These poor and variable results may be due to the lack of a standard CT criteria for diagnosing metastatic lymph nodes. Various criteria used for malignant lymph nodes are those with short axis diameters of 6-8 $\mathrm{mm}$ for perigastric lymph nodes, roundness and central necrosis, heterogeneous enhancement, more than $1 \mathrm{~cm}$ without fatty hilum, marked enhancement (over 80 or $100 \mathrm{HU}$ ), and clustering of more than three lymph nodes.(22) Although there is a clear correlation between the lymph node size and metastasis, microscopic nodal metastases in normal-size lymph nodes and lymph node enlargement resulting from reactive or inflammatory change are common. Microscopic metastases can frequently be found in normal-sized lymph nodes of patients which makes accurate $\mathrm{N}$-staging more difficult. In our study, sensitivity, specificity and accuracy of N0 staging was $73 \%, 85 \% 80 \%$, N1 was $72.7 \%, 75 \%$ and $74 \%$, and for $\mathrm{N} 2$ it was $71 \%, 92.9 \%$ and $88.5 \%$ respectively. Out of the 14 cases detected to have N0 staging, 11 were histopathologically proved. There were 3 false positives and 4 false negatives. Out of the 14 cases detected to be in N1 staging, 11 cases were proved. There were 6 false positive and 3 false negatives. There were 7 cases which were under N2 stage according to CT, 5 cases were proved. There were 2 false positives and 2 false negatives. The study by Chen et al had shown similar result with sensitivity, specificity and accuracy between 71\%- 81\%, 79\% - 94\% and 80\% - 91\% respectively.

To summarise, imaging of early gastric cancer with MDCT poses challenges. Ability to differentiate between T1 and T2 lesions is not very high. Attention to details of technique like achieving adequate distension and use of negative contrast and adequate IV contrast with reformatting of images in a dedicated workstation are essential to achieve acceptable results. Endoscopic ultrasound will probably remain the primary modality to assess the tumour depth. However, for more advanced cases, CT is definitely the modality of choice. 
Our results show high sensitivity and specificity in accurate staging and in the detection of advanced cases.

\section{Study Limitations}

We used positive oral contrast medium for the CECT evaluation as our study design included both suspected and incidentally detected cases. Another issue was lack of proper distension of stomach in some cases which made evaluating T1 lesions difficult.

\section{CONCLUSION}

Based on current findings it can be concluded that routinely used protocol for MDCT have high sensitivity, specificity and accuracy in staging gastric adenocarcinomas. These parameters are lower when staging is done for T1 lesions (55.6\%, 96.2\% and $85.7 \%$ ) respectively as compared to T3 and $\mathrm{T} 4$ lesions $(93.8 \%, 84.2 \%, 88 \%)$ and $(66 \%, 100 \%$ and 91\%) respectively. For nodal staging our study revealed a high sensitivity, specificity and accuracy, with sensitivity varying from $71 \%$ to $73 \%$, specificity varying from $75 \%$ to $92 \%$ and accuracy varying from $74 \%$ to $88 \%$.

\section{REFERENCES}

[1] Angelelli G, Ianora AA, Scardapane A, et al. Role of computerized tomography in the staging of gastrointestinal neoplasms. Semin Surg Oncol 2001;20(2):109-21.

[2] Ba-Ssalamah A, Prokop M, Uffmann M, et al. Dedicated multidetector CT of the stomach: spectrum of diseases. Radiogr 2003;23(3):625-44.

[3] Habermann CR, Weiss F, Riecken R, et al. Preoperative staging of gastric adenocarcinoma: comparison of helical CT and endoscopic US. Radiology 2004;230(2):465-71.

[4] Brinton LA, Gridley G, Hrubec Z, et al. Cancer risk following pernicious anaemia. $\mathrm{Br} \mathrm{J}$ Cancer 1989;59(5):810-3.

[5] Fox JG, Wang TC. Inflammation, atrophy and gastric cancer. J Clin Invest 2007;117(1):60-9.

[6] Mirvish SS. Effects of vitamins C and E on N-nitroso compound formation, carcinogenesis and cancer. Cancer 1986;58(8 Suppl):1842-50.

[7] Correa P, Houghton J. Carcinogenesis of Helicobacter pylori. Gastroenterology 2007;133(2):659-72.

[8] Morales TG. Adenocarcinoma of the gastric cardia. Dig Dis Basel Switz 1997;15(6):346-56.

[9] Olearchyk AS. Gastric carcinoma. A critical review of 243 cases. Am J Gastroenterol 1978;70(1):25-45.

[10] Cappell MS, Friedel D. The role of esophagogastroduodenoscopy in the diagnosis and management of upper gastrointestinal disorders. Med Clin North Am 2002;86(6):1165-216.
[11] Low VH, Levine MS, Rubesin SE, et al. Diagnosis of gastric carcinoma: sensitivity of double-contrast barium studies. AJR Am J Roentgenol 1994;162(2):329-34.

[12] Chen CY, Hsu JS, Wu DC, et al. Gastric cancer: preoperative local staging with 3D multi-detector row CT--correlation with surgical and histopathologic results. Radiology 2007;242(2):472-82.

[13] Kim YH, Lee KH, Park SH, et al. Staging of T3 and T4 gastric carcinoma with multidetector CT: added value of multiplanar reformations for prediction of adjacent organ invasion. Radiology 2009;250(3):767-75.

[14] Kim HJ, Kim AY, Oh ST, et al. Gastric cancer staging at multi-detector row CT gastrography: comparison of transverse and volumetric CT scanning. Radiology 2005;236(3):879-85.

[15] Gore RM. Gastric cancer. Clinical and pathologic features. Radiol Clin North Am 1997;35(2):295-310.

[16] Kim JP. Surgical results in gastric cancer. Semin Surg Oncol 1999;17(2):132-8.

[17] Yeole BB. Trends in cancer incidence in esophagus, stomach, colon, rectum and liver in males in India. Asian Pac J Cancer Prev 2008;9(1):97-100.

[18] Jemal A, Bray F, Center MM, et al. Global cancer statistics. CA Cancer J Clin 2011;61(2):69-90.

[19] Thiruvananthapuram_Ann.pdf http://ncrpindia.org/ALL_NCRP_REPORTS/PBCR_REPO RT_2012_2014/ALL_CONTENT/Annexure/Thiruvanant hapuram_Ann.pdf

[20] Kollam_Ann.pdf http://ncrpindia.org/ALL_NCRP_REPORTS/PBCR_REPO RT_2012_2014/ALL_CONTENT/Annexure/Kollam_Ann. pdf

[21] Minami M, Kawauchi N, Itai Y, et al. Gastric tumours: radiologic-pathologic correlation and accuracy of $\mathrm{T}$ staging with dynamic CT. Radiology 1992;185(1):173-8.

[22] Choi JI, Joo I, Lee JM. State-of-the-art preoperative staging of gastric cancer by MDCT and magnetic resonance imaging. World J Gastroenterol 2014;20(16):4546-57.

[23] Lee MH, Choi D, Park MJ, et al. Gastric cancer: imaging and staging with MDCT based on the $7^{\text {th }}$ AJCC guidelines. Abdom Imaging 2012;37(4):531-40.

[24] Kim AY, Kim HJ, Ha HK. Gastric cancer by multidetector row CT: preoperative staging. Abdom Imaging 2005;30(4):465-72.

[25] Shimizu K, Ito K, Matsunaga N, et al. Diagnosis of gastric cancer with MDCT using the water-filling method and multiplanar reconstruction: CT-histologic correlation. Am J Roentgenol 2005;185(5):1152-8.

[26] Kwee RM, Kwee TC. Imaging in assessing lymph node status in gastric cancer. Gastric Cancer 2009;12(1):6-22. 